\title{
Granulocyte-colony stimulating factor levels in bronchoalveolar lavage fluid from patients with idiopathic pulmonary fibrosis
}

Jun-ichi Ashitani, Hiroshi Mukae, Haruko Taniguchi, Toshihiko Ihi, Jun-ichi Kadota, Shigeru Kohno, Shigeru Matsukura

\begin{abstract}
Background-Granulocyte-colony stimulating factor (G-CSF) is known as a potent neutrophil chemotactic glycoprotein in vitro but its contribution to chemotactic activity in neutrophil mediated lung diseases is not yet known. The aims of this study were to determine whether G-CSF is present in high concentrations in bronchoalveolar lavage (BAL) fluid of patients with idiopathic pulmonary fibrosis (IPF, also called cryptogenic fibrosing alveolitis), a neutrophil mediated lung disease, and to what extent G-CSF in BAL fluid contributes to neutrophil accumulation in the lung of patients with IPF.
\end{abstract}

Methods-G-CSF concentrations in BAL fluid samples from 16 healthy volunteers, 24 patients with IPF, and 73 patients with non-IPF lung disease were measured by enzyme linked immunosorbent assay. The relationship between G-CSF concentrations and neutrophil count in BAL fluid was also examined. Neutrophil chemotactic activity (NCA) was measured in BAL fluid in healthy volunteers and patients with IPF. The contribution of G-CSF to overall NCA in lungs with IPF was assessed by repeating the measurement of NCA after a complete neutralisation of G-CSF bioactivity by anti-human G-CSF antiserum.

Results-Detectable levels of G-CSF were found in BAL fluid of $83 \%$ of patients with IPF while the levels in all healthy volunteers were below the detection limit. In patients with IPF a significant correlation was observed between the BAL fluid neutrophil count and the concentration of G-CSF in the BAL fluid. The neutrophil count also correlated significantly with percentage forced vital capacity. In BAL fluid samples from patients with IPF the mean NCA value was reduced by $35 \%$ after neutralisation with an anti-human G-CSF antiserum.

Conclusions-G-CSF may be involved in enhancing neutrophil accumulation in the lungs of patients with IPF.

(Thorax 1999;54:1015-1020)

Keywords: granulocyte-colony stimulating factor; bronchoalveolar lavage fluid; idiopathic pulmonary fibrosis
Idiopathic pulmonary fibrosis (IPF), also known as cryptogenic fibrosing alveolitis, is a chronic progressive disorder of the lung parenchyma characterised by both inflammation and fibrosis. ${ }^{1}$ Several studies have shown that neutrophils, ${ }^{23}$ in addition to eosinophils, ${ }^{4}$ contribute to the pathogenesis of IPF and increased neutrophil numbers in bronchoalveolar lavage (BAL) fluid are associated with extensive disease. ${ }^{56}$ However, the mechanisms leading to persistent accumulation of neutrophils in the lung of these patients are not well understood. It is likely that several neutrophil chemotactic factors, such as interleukin (IL)-8, leukotrienes, and granulocyte colony stimulating factor (G-CSF), are released in the lower respiratory tract, attracting neutrophils from the pulmonary capillary blood into the parenchyma. $^{7-10}$

G-CSF, a $20 \mathrm{kD}$ glycoprotein, is known to induce granulopoiesis in vitro and in vivo and to enhance a variety of functions of mature neutrophils. ${ }^{11-16}$ This cytokine has been shown to be a potent neutrophil chemotactic factor ${ }^{17}$ and to enhance the expression of adhesion molecules on neutrophils. ${ }^{18}$ These findings suggest that G-CSF may play an important part in the pathogenesis of neutrophil mediated pulmonary disease including IPF. However, to our knowledge, there are no studies that have examined the presence of G-CSF in BAL fluid of patients with neutrophil mediated pulmonary diseases.

We therefore measured BAL fluid concentrations of G-CSF in patients with various pulmonary diseases including IPF, and investigated whether G-CSF in the BAL fluid of patients with IPF has neutrophil chemotactic activity. We also investigated the relationship between those concentrations and clinical data in patients with IPF to determine the role of G-CSF in neutrophil accumulation in the lung of these patients.

\section{Methods}

STUDY POPULATION

BAL fluid samples were collected from 16 healthy volunteers (10 men) of median age 28 years (range 19-64), 11 of whom were non-smokers and five were current smokers, and 97 patients with pulmonary diseases ( 57 men) of median age 58 years (range 22-79), 38 of whom were non-smokers, 43 ex-smokers, and 16 current smokers. These included 24 patients with IPF (12 men) of median age 63 years (range 41-78), 15 of whom were 
Table 1 Diagnoses of patients studied $(n=113)$

\begin{tabular}{lc}
\hline Healthy volunteers & 16 \\
Idiopathic pulmonary fibrosis & 24 \\
Diffuse panbronchiolitis & 10 \\
Sarcoidosis & 11 \\
Bronchiolitis obliterans organizing pneumonia & 7 \\
Hypersensitivity pneumonitis & 4 \\
Collagen vascular disease & 7 \\
Rheumatoid arthritis & 4 \\
Progressive systemic sclerosis & 2 \\
CREST syndrome & 1 \\
Wegener's granulomatosis & 2 \\
Bacterial pneumonia & 4 \\
Non-bacterial pneumonia & 7 \\
Pneumocystis carinii & 2 \\
Mycobacterium tuberculosis & 2 \\
Atypical mycobactelosis & 1 \\
Parasite & 1 \\
Mycoplasma & 1 \\
Bronchial asthma & 4 \\
Bronchiectasis & 6 \\
Acute respiratory distress syndrome & 9 \\
Others & 2 \\
Histiocytosis & 1 \\
Alveolar proteinosis & 1 \\
\hline
\end{tabular}

non-smokers, eight ex-smokers, and one current smoker, and 73 disease controls (table 1). The diagnosis of IPF and bronchiolitis obliterans organising pneumonia (BOOP) was pathologically confirmed by open lung biopsy or by necropsy. The diagnosis of sarcoidosis was based on pathological examination of transbronchial lung biopsy (TBLB) specimens. The diagnosis of collagen vascular disease was established according to the criteria for the respective diseases and the presence of diffuse interstitial opacities on the chest radiograph. The respective pulmonary infections were confirmed by sputum culture or pathologically by TBLB. Diagnosis of asthma was established on the basis of a clinical history of wheezing and bronchial hypersensitivity to methacholine. Pulmonary function tests and arterial blood gas analysis were performed in all patients with IPF prior to BAL. None of these patients had received corticosteroids, antibiotics, or immunosuppressive agents during the year before BAL examination. BAL was also performed before treatment in patients with other lung diseases.

BRONCHOALVEOLAR LAVAGE

With the informed consent of all patients, BAL was performed according to the ERS guidelines ${ }^{19}$ using a flexible fibreoptic bronchoscope (Olympus, P-20 Olympus, Tokyo, Japan) after local anaesthesia of the upper airway with $2 \%$ lidocaine. The bronchoscope was wedged into one of the segmental bronchi of the right middle lobe for lavage and an aliquot of $50 \mathrm{ml}$ sterile saline at body temperature was instilled through the bronchoscope. The fluid was immediately retrieved by gentle suction using a sterile syringe. The entire procedure of instillation and retrieval was repeated three times. BAL fluid was passed through two sheets of gauze and then centrifuged at $500 \mathrm{~g}$ for $10 \mathrm{~min}-$ utes at $4^{\circ} \mathrm{C}$. The remaining fluid was centrifuged at $500 \mathrm{~g}$ for five minutes and supernatant was stored at $-80^{\circ} \mathrm{C}$ until examined. After washing twice with phosphate buffered saline free of calcium or magnesium (PBS, Gibco, UK), the remaining cells were suspended in PBS supplemented with $10 \%$ heat inactivated fetal calf serum and counted using a haemocytometer. The aliquot was then diluted to a concentration of $2 \times 10^{5}$ cells $/ \mathrm{ml}$ and $0.2 \mathrm{ml}$ cell suspension was spun down onto a glass slide at $1100 \mathrm{rpm}$ for two minutes using a Cytospin 2 cytocentrifuge (Shandon Instruments, Sewickley, Pennsylvania, USA). The resulting slides were dried, fixed, and then stained using a May-Giemsa method. Differential cell counts were determined from more than 200 cells using a photomicroscope.

MEASUREMENT OF G-CSF IN BAL FLUID

G-CSF was measured by the enzyme linked immunosorbent assay (ELISA) kit (Amersham, Tokyo, Japan) using the quantitative immunometric "sandwich" enzyme immunoassay technique. Samples (supernatant) were pipetted into wells coated with a monoclonal antibody specific for G-CSF. An enzyme linked polyclonal antibody specific for G-CSF was added to the wells and allowed to bind to G-CSF which was bound during the first incubation. In the next step a substrate solution was added to the wells and the developed colour was proportional to the amount of G-CSF in the initial step. Colour development was terminated by the addition of stop solution and the intensity of the colour was measured. The lower limit of detection was $15.6 \mathrm{pg} / \mathrm{ml}$.

\section{MEASUREMENT OF G-CSF IN SERUM}

Serum G-CSF concentrations were also measured in healthy controls and patients with IPF using ELISA. We could not measure serum G-CSF concentrations in patients with non-IPF lung diseases because this study was performed on stored samples and serum was not available in patients with non-IPF lung diseases.

RELATIONSHIP BETWEEN NEUTROPHIL COUNT IN BAL FLUID AND PULMONARY FUNCTION

Pulmonary function tests including percentage forced vital capacity (FVC), carbon monoxide transfer factor (TLCO), and arterial blood gas analysis were performed in all patients with IPF before BAL was performed or treatment commenced. Statistical analyses were made between the neutrophil count in the BAL fluid and the results of pulmonary function tests or arterial blood gas analysis in IPF patients.

RELATIONSHIP BETWEEN NEUTROPHIL COUNT OR PULMONARY FUNCTION AND G-CSF IN BAL FLUID Based on the concentrations of G-CSF measured in BAL fluid, statistical analyses were made between the neutrophil count in BAL fluid or the results of pulmonary function tests and G-CSF concentrations in BAL fluid in patients with IPF to evaluate whether G-CSF participated in neutrophil influx into the lung or deterioration of pulmonary function in these patients.

NEUTROPHIL CHEMOTACTIC ASSAY

Neutrophil chemotactic activity (NCA) in BAL fluid was measured in healthy controls and patients with IPF using a 48 well microchemotaxis chamber (Neuroprobe Inc, 


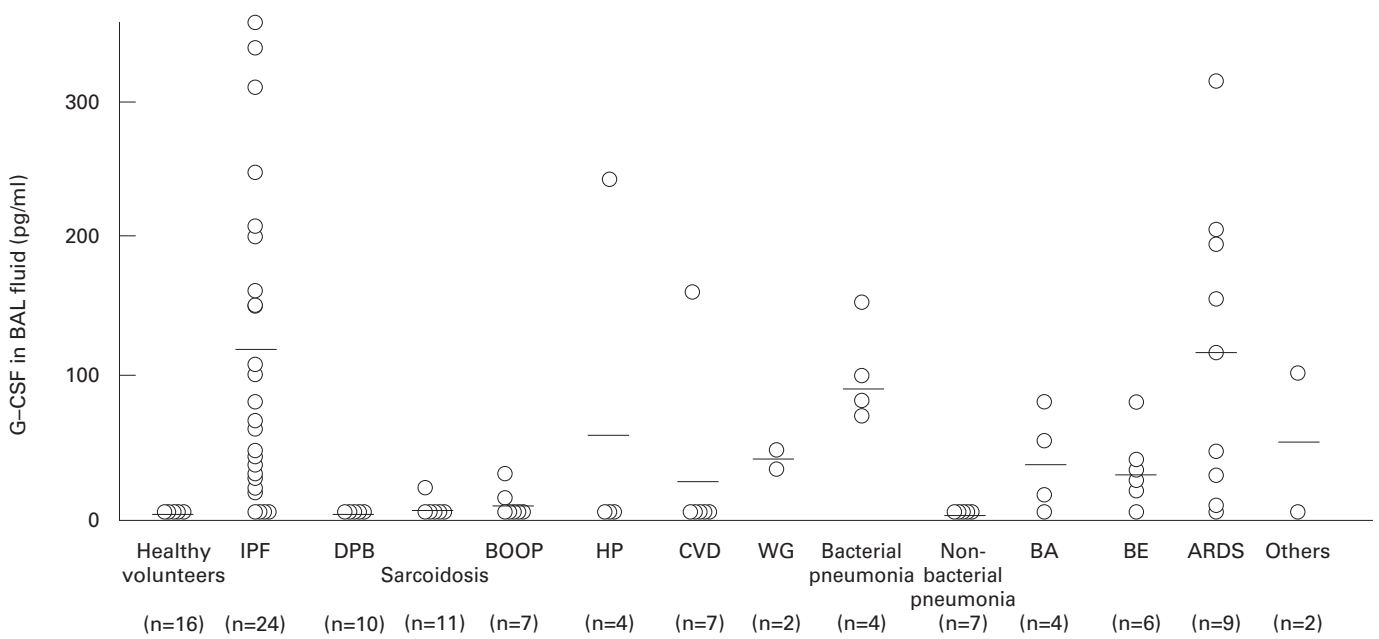

Figure 1 Individual G-CSF levels in BAL fluid of normal healthy volunteers and patients with various pulmonary diseases. Means indicated by horizontal bars. IPF = idiopathic pulmonary fibrosis; DPB = diffuse panbronchiolitis; BOOP $=$ bronchiolitis obliterans organising pneumonia; $H P=$ hypersensitivity pneumonitis; $C V D=$ collagen vascular disease; $W G=$ Wegener's granulomatosis, $B A=$ bronchial asthma; $B E=$ bronchiectasis; $A R D S=$ acute respiratory distress syndrome.

Bethesda, Maryland, USA) as described by Falk et al. ${ }^{20}$ Twenty five $\mathrm{ml}$ aliquot samples were added to the bottom wells and a polycarbonate filter sheet ( $3 \mathrm{~mm}$ pores, NFB3-PVPF, Costar Corp, Cambridge, Massachusetts, USA) was placed on the top of the wells in the bottom plate. The neutrophils (50 ml aliquots) obtained from healthy subjects as described by Ferrante and Thong ${ }^{21}$ were placed in the upper wells at a concentration of $3 \times 10^{6}$ cells $/ \mathrm{ml}$ in Hank's solution with $0.1 \%$ BSA. Chambers were incubated for 30 minutes at $37^{\circ} \mathrm{C}$ in a humidified atmosphere of $95 \%$ air $/ 5 \% \mathrm{CO}_{2}$; the filters were removed, fixed in absolute methanol, and stained with eosin-azur (DiffQuik, Harleco, Gibbstown, New Jersey, USA). Cells that migrated through the filter to the other side were counted. NCA was measured as the mean number of cells per 10 high power fields $(\times 1000)$. The results were expressed as a percentage of the chemotactic response to $10^{-7} \mathrm{M}$ N-formyl-Met-Leu-Phe (FMLP; Sigma Chemicals, St Louis, Missouri, USA). In each experiment a negative control was assessed using Hank's solution.

The contribution of G-CSF to overall NCA in the BAL fluid of patients with IPF was assessed by repeating the measurement of NCA after a complete neutralisation of G-CSF bioactivity with anti-human G-CSF antiserum (Chugai, Tokyo). Samples of BAL fluid were incubated with anti-human G-CSF antibody for 30 minutes at $37^{\circ} \mathrm{C}$. The mixture was then centrifuged at $7000 \mathrm{~g}$ for 10 minutes and the supernatant was obtained for measurement of NCA. The NCA was inhibited dose dependently by previous anti-human G-CSF antiserum $\left(1: 10^{3}\right.$ to $1: 10^{6}$ dilution). Samples were mixed with $1: 10^{3}$ diluted antihuman G-CSF antiserum which resulted in a complete inhibition of NCA induced by $200 \mathrm{pg} / \mathrm{ml} \mathrm{G-CSF}{ }^{22}$

STATISTICAL ANALYSIS

Data were expressed as mean (SE). Differences between groups were examined using the Mann-Whitney U test. Correlations between the neutrophil count in BAL fluid and clinical data or G-CSF concentrations in BAL fluid were determined using the Spearman's rank correlation analysis. For comparison of NCA before and after neutralisation of G-CSF in BAL fluid a paired samples Wilcoxon test was used to determine statistical significance. A p value of $<0.05$ denoted the presence of a statistically significant difference.

\section{Results}

BAL FLUID AND SERUM G-CSF CONCENTRATIONS G-CSF concentrations in the BAL fluid of 16 healthy volunteers were below the level of detection. The proportion of BAL fluid samples with detectable levels of G-CSF was $49.5 \%(48 / 97)$ in the entire group of patients. The mean BAL fluid G-CSF concentration in patients with IPF was $120 \mathrm{pg} / \mathrm{ml}$ (range 0-360, fig 1 ). There was no significant difference in the G-CSF concentration in the BAL fluid of smokers and non-smokers (data not shown). BAL fluid G-CSF concentrations were detectable in a proportion of patients with sarcoidosis $(1 / 11$, mean $6 \mathrm{pg} / \mathrm{ml})$, BOOP $(2 / 7$, mean $9 \mathrm{pg} / \mathrm{ml})$, hypersensitivity pneumonitis (1/4, mean $60 \mathrm{pg} / \mathrm{ml})$, collagen vascular disease $(1 / 7$, mean $24 \mathrm{pg} / \mathrm{ml}$ ), Wegener's granulomatosis $(2 / 2$, mean $45 \mathrm{pg} / \mathrm{ml})$, bacterial pneumonia (4/4, mean $110 \mathrm{pg} / \mathrm{ml})$, asthma (3/4, mean $40 \mathrm{pg} / \mathrm{ml}$ ), bronchiectasis (5/6, mean $37 \mathrm{pg} /$ $\mathrm{ml}$ ), and acute respiratory distress syndrome (8/9, median $124 \mathrm{pg} / \mathrm{ml})$.

Serum G-CSF concentrations were detectable only in two of 24 patients with IPF and not in healthy volunteers.

RELATIONSHIP BETWEEN BAL FLUID NEUTROPHIL COUNTS AND PULMONARY FUNCTION OR ARTERIAL BLOOD GAS TENSIONS IN PATIENTS WITH IPF

The proportion of neutrophils, alveolar macrophages, lymphocytes, and eosinophils in the BAL fluid of patients with IPF was $8 \%, 77 \%$, $14 \%$, and $1 \%$, respectively. Mean (SE) BAL fluid total cell count in patients with IPF (242 $(31) \times 10^{3} / \mathrm{ml}$ ) was significantly higher than in healthy volunteers $\left(132(22) \times 10^{3} / \mathrm{ml}\right.$, table 2$)$. 
Table 2 Mean (SE) BAL fluid findings in healthy volunteers and patients with IPF

\begin{tabular}{lll}
\hline & $\begin{array}{l}\text { Healthy } \\
\text { volunteers } \\
(n=16)\end{array}$ & IPF $(n=24)$ \\
\hline Total cell $\left(\times 10^{3} / \mathrm{ml}\right)$ & $132(22)$ & $242(31)^{\star}$ \\
Macrophages $\left(\times 10^{3} / \mathrm{ml}\right)$ & $114(2)$ & $186(7)^{\star}$ \\
Macrophages $(\%)$ & $86(1)$ & $77(3)^{\star}$ \\
Lymphocytes $\left(\times 10^{3} / \mathrm{ml}\right)$ & $15(1)$ & $34(5)^{\star}$ \\
Lymphocytes $(\%)$ & $11(1)$ & $14(2)$ \\
Neutrophils $\left(\times 10^{3} / \mathrm{ml}\right)$ & $3(1)$ & $4(1)^{\star}$ \\
Neutrophils $(\%)$ & $2(1)$ & $8(2)^{\star}$ \\
Eosinophils $\left(\times 10^{3} / \mathrm{ml}\right)$ & $1(1)$ & $4(1)^{\star}$ \\
Eosinophils $(\%)$ & $1(1)$ & $1(1)$ \\
\end{tabular}

Value are mean (SE).

${ }^{\star} \mathrm{p}<0.05$ vs healthy volunteers.

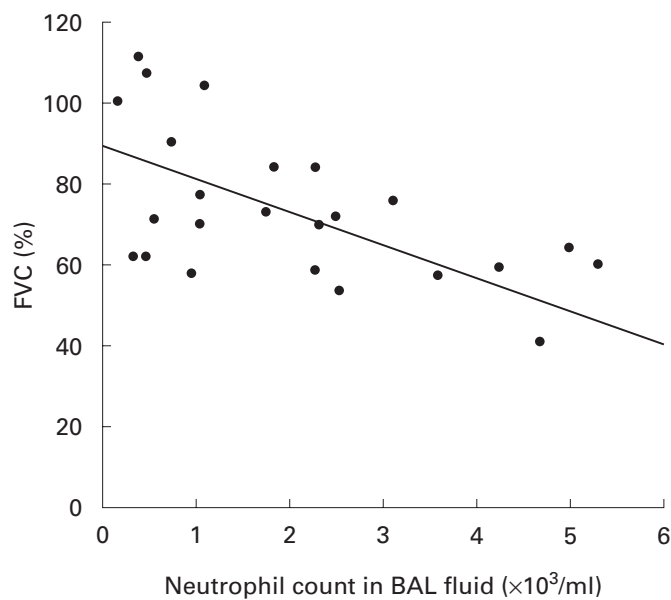

Figure 2 Relationship between \% forced vital capacity (FVC) and BAL fluid neutrophil count in patients with $I P F$.

The BAL fluid neutrophil count in patients with IPF was also significantly higher than in healthy volunteers. The mean (SE) FVC, TLCO, and $\mathrm{PaO}_{2}$ in patients with IPF were 73.9 (3.6) $\%, 46.9$ (3.5) \% and $10.4(0.4) \mathrm{mm} \mathrm{Hg}$, respectively. The BAL fluid neutrophil count in patients with IPF correlated inversely with $\%$ FVC $(r=-0.58, \mathrm{p}<0.005$, fig 2$)$ but not with $\%$ TLCO or $\mathrm{PaO}_{2}$ (data not shown).

RELATIONSHIP BETWEEN NEUTROPHIL COUNT AND BAL FLUID CONCENTRATIONS OF G-CSF IN PATIENTS WITH IPF

There was a significant correlation between BAL fluid G-CSF concentrations and absolute neutrophil counts $(r=0.64, \mathrm{p}<0.001$, fig 3$)$ in patients with IPF, but no such relationship was present with percentage neutrophil count. These results suggest that G-CSF concentrations in the BAL fluid might be involved in accumulation of neutrophils in the lungs of patients with IPF.

RELATIONSHIP BETWEEN PULMONARY FUNCTION AND BAL FLUID CONCENTRATIONS OF G-CSF IN PATIENTS WITH IPF

There was no significant correlation between the concentration of G-CSF in the BAL fluid and \%FVC, \% TLCO, or $\mathrm{PaO}_{2}$ in patients with IPF.

NEUTROPHIL CHEMOTACTIC ACTIVITY (NCA)

Figure 4 shows changes in NCA before and after neutralisation of G-CSF bioactivity with anti-human G-CSF antiserum in BAL fluid of

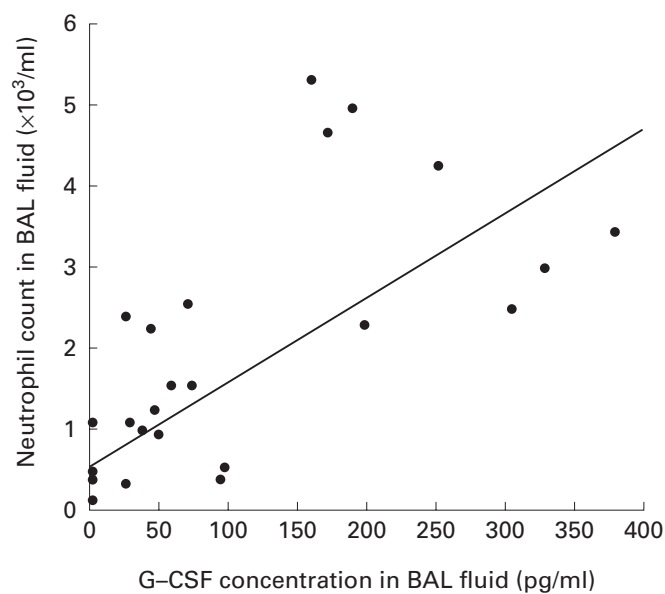

Figure 3 Relationship between neutrophil count and concentration of G-CSF in BAL fluid of patients with IPF.

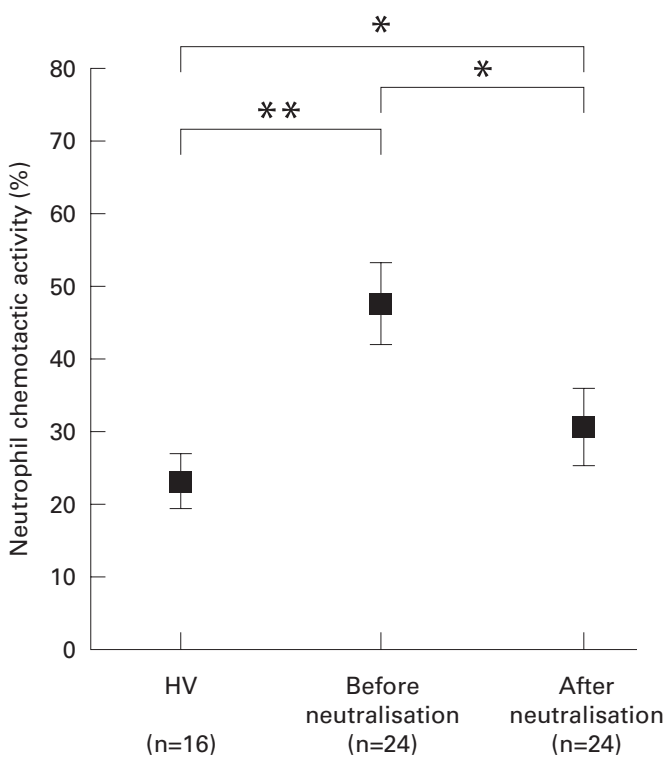

Figure 4 Neutrophil chemotactic activity in BAL fluid of healthy volunteers $(H V)$ and patients with IPF before and after neutralisation of G-CSF bioactivity with anti-human $G-C S F$ antibody. ${ }^{\star} p<0.05 ;{ }^{\star} *<0.01$. Closed squares represent means and $S E$ values are indicated by the horizontal bars.

patients with IPF. The median NCA value before neutralisation was 48.5 (4.2)\% which decreased after neutralisation to 31.6 (3.6)\%. NCA values after neutralisation were also higher than those of healthy volunteers. These findings indicate that G-CSF in the lungs of patients with IPF is a potent neutrophil chemotactic factor in vitro.

\section{Discussion}

The fact that neutrophils constitute a significant component of alveolar inflammatory cells in IPF has been confirmed by several methods including examination of BAL fluid and isolation of inflammatory cells from open lung biopsy specimens. ${ }^{2}{ }^{35}$ Adherence to the endothelium and migration into the tissue are two essential steps during the process of neutrophil accumulation at inflammatory sites. Recent studies have shown the effects of G-CSF on enhanced expression of adhesion molecules ${ }^{18}$ and chemotaxis ${ }^{17}$ on human neutrophils. Because G-CSF is a potent activator of mature neutrophils and enhances release of 
superoxide, activation of neutrophils by G-CSF in the lung may cause deterioration of lung function through the action of superoxides and neutrophil products. However, G-CSF levels in BAL fluid did not correlate with FVC or TLCO in patients with IPF in the present study. Wells et al reported that neutrophils are linked to the morphological extent of disease in patients with IPF and eosinophils are more closely linked to functional impairment than neutrophils. ${ }^{4}$ The lack of correlation between G-CSF levels in BAL fluid and $\%$ FVC or TLCo in patients with IPF may be supported by the fact that G-CSF activates neutrophils but not eosinophils, although both eosinophils and neutrophils play a major part in the pathogenesis of IPF.

IL-8 is known as a potent chemoattractant for neutrophils and its contribution to neutrophil influx into the lung has been demonstrated in diffuse panbronchiolitis, ${ }^{23}$ acute respiratory distress syndrome, ${ }^{24}$ and IPF. ${ }^{25}$ Carrè et al reported the presence of increased levels of IL-8 mRNA in patients with IPF and those with collagen vascular disease with pulmonary fibrosis, which was correlated with the number of neutrophils in the BAL fluid. ${ }^{10}$ These results were further expanded by the same group ${ }^{26}$ who showed that other neutrophil chemotaxins, in addition to IL-8, might be present in the BAL fluid of patients with IPF, based on higher neutrophil chemotaxis relative to the values of IL-8. In the present study the BAL fluid of patients with IPF was capable of attracting neutrophils and is likely to have contained several other neutrophil chemotactic factors. Although the use of anti-G-CSF antiserum resulted in some inhibition of neutrophil chemotaxis in the BAL fluid of patients with IPF, the antibody did not produce complete inhibition of neutrophil migration. This finding suggests the presence of another chemoattractant for neutrophils in addition to G-CSF.

Tazi et $a l^{77}$ reported that lavage cells in certain interstitial lung diseases spontaneously produced G-CSF but that G-CSF was not detected in normal controls. Our results are in part consistent with these findings. Moreover, we also detected G-CSF in the BAL fluid of patients with Wegener's granulomatosis, bacterial pneumonia, acute respiratory distress syndrome, and a proportion of those with collagen vascular disease and asthma. G-CSF induced neutrophil chemoattraction and activation, which may be immunologically advantageous in bacterial pneumonia but deleterious in Wegener's granulomatosis, acute respiratory distress syndrome, collagen vascular disease, and asthma. Diffuse panbronchiolitis is also a neutrophil related pulmonary disease and some studies have indicated that IL-8 may play a part in neutrophil accumulation in the respiratory tract of patients with this disease. ${ }^{23}$ However, our results showed that G-CSF concentrations in the BAL fluid of patients with diffuse panbronchiolitis were all below the level of detection. It is possible that there may be some differences in the type of neutrophil chemoattractant factors involved in the pathologi- cal process among various neutrophil related pulmonary diseases.

Plasma levels of G-CSF in all but two patients with IPF were below the detectable level. Although high plasma G-CSF concentrations were reported in patients with infection, ${ }^{28}$ these two patients were not considered as cases with pulmonary infection because of negative $\mathrm{C}$ reactive protein (CRP) and normal blood neutrophil count. The detectable G-CSF concentration in the serum of these cases might be caused by leakage of this factor from the respiratory tract into the bloodstream. Our results could not establish the exact contributory role of G-CSF in neutrophil recruitment into the lung of patients with acute respiratory distress syndrome because of the small sample size. Two patients with collagen vascular disease showed detectable BAL fluid G-CSF concentrations. One of these had a high percentage of neutrophils in the BAL fluid and also bilateral basal pulmonary fibrosis on radiological examination.

In conclusion, G-CSF may enhance the accumulation of neutrophils in the lung of patients with IPF. Further studies are necessary to clarify whether G-CSF participates in the pathogenesis of neutrophil accumulation in IPF and other neutrophil mediated lung diseases such as acute respiratory distress syndrome and collagen vascular disease.

1 Crystal RG, Gadek JE, Ferrans JD, et al. Interstitial lung disease: current concepts of pathogenesis, staging and therapy. Am 7 Med 1981;70:542-68.

2 Haellgren R, Bjermer L, Lundgren R, et al. The eosinophil component of the alveolitis in idiopathic pulmonary fibrosis. Signs of eosinophil activation in the lung are related to impaired lung function. Am Rev Respir Dis 1989;139:373-7.

Gadek JE, Hunninghake GW, Zimmermann RL, et al. Regulation of the release of alveolar macrophage-derived neutrophil chemotactic factor. Am Rev Respir Dis 1980, 121:723-33

4 Wells AU, Hansell DM, Haslam PL, et al. Bronchoalveolar lavage cellularity: lone cryptogenic fibrosing alveolitis compared with the fibrosing alveolitis of systemic sclerosis. $A m$ F Respir Crit Care Med 1998;157:1474-82.

5 Rudd RM, Haslam PL, Turner-Warwick M. Cryptogenic fibrosing alveolitis. Relationships of pulmonary physiology and bronchoalveolar lavage to response to treatment and prognosis. Am Rev Respir Dis 1981;124:1-8.

6 Wells AU, Hansell DM, Rubens MB, et al. Fibrosing alveolitis in systemic sclerosis: bronchoalveolar lavage findings in relation to computed tomographic appearance. Am f Respir Crit Care Med 1994;150:462-8.

7 Ozaki T, Hayashi H, Tani K, et al. Neutrophil chemotactic factors in the respiratory tract of patients with chronic airway diseases or idiopathic pulmonary fibrosis. Am Rev Respir Dis 1992;145:85-91.

8 Car BD, Meloni F, Luisetti M, et al. Elevated IL-8 and $\mathrm{MCP}-1$ in the bronchoalveolar lavage fluid of patients with idiopathic pulmonary fibrosis and pulmonary sarcoidosis. Am $\mathcal{~}$ Respir Crit Care Med 1994;149:655-9.

9 Xaubet A, Agusti C, Luburich P, et al. Interleukin-8 expression in bronchoalveolar lavage cells in the evaluation of alveolitis in idiopathic pulmonary fibrosis. Respir Med 1998;92:338-44.

10 Carrè PC, Mortenson RL, King TE Jr, et al. Increased expression of the interleukin-8 gene by alveolar macrophages in idiopathic pulmonary fibrosis. 7 Clin Invest 1991; ph:1802-10.

11 Metcalf D. The molecular biology and functions of the granulocyte-macrophage colony stimulating factors. Blood 1986;67:257-67.

12 Platzer E, Welte K, Gabrilove JL, et al. Biological activities of a human pluripotent hemopoietic colony-stimulating factor on normal and leukemic cells. $\mathcal{F}$ Exp Med 1985;162: $1788-801$

13 Cohen AM, Zsebo $\mathrm{KM}$, Inoue $\mathrm{H}$, et al. In vivo stimulation of granulopoiesis by recombinant human granulocyte colony-

14 Souza LM Boone TC Gabrilove J, et al. Recombinant Souza LM, Boone TC, Gabrilove J, et al. Recombinant human granulocyte-colony stimulating factor: effects on

15 Welte K, Bonilla MA, Gillio AP, et al. Recombinant human granulocyte-colony stimulating factor: effects on hematopoiesis in normal and cyclophosphamide-treated primates. F Exp Med 1987;165:941-9. 
16 Gabrilove JL, Jakubowski A, Scher H, et al. Effects of granulocyte colony-stimulating factor on neutropenia and associated morbidity due to chemotherapy for transitional cell carcinoma of the urothelium. N Engl F Med 1988;318: 1414-22.

17 Wang JM, Chen ZG, Colella S, et al. Chemotactic activity of recombinant human granulocyte colony-stimulating factor. Blood 1988;72:1456-60.

18 Yong KL, Linch DC. Differential effects of granulocyte- and and GM-CSF) on neutropil adhesion in vitro and in vivo. Eur 7 Haematol 1992;49:251-9.

19 Klech H, Pohl W. Technical recommendations and guidelines for bronchoalveolar lavage (BAL). Eur Respir f 1989; 2:561-85.

20 Falk W, Goodwin RH, Leonard EJ. A 48-well microchemotaxis assembly for rapid and accurate measurement of leu-

21 Ferrante A, Thong YH. A rapid one-step procedure for purification of mononuclear and polymorphonuclear leukocytes from human blood using a modification of the hypaque-ficoll technique. F Immunol Methods 1978;24: 389-93.

22 Kamachi S, Matsubara K, Kobayashi T, et al. Preparation and characteristics of anti-recombinant human G-CSF (rGCSF) antibodies. Fpn Pharmacol Ther 1990;18: S2221-6.
23 Oishi K, Sonoda F, Kobayashi S, et al. Role of interleukin-8 (IL-8) and an inhibitory effect of erythromycin on IL-8 release in the airways of patients with chronic airway diseases. Infect Immun 1994;62:4145-52.

24 Miller EJ, Cohen AB, Nagao S, et al. Elevated levels of NAP-1/interleukin-8 are present in the airspaces of patients with the adult respiratory distress syndrome and are associated with increased mortality. Am Rev Respir Dis 1992;146: 427-32.

25 Ozaki T, Hayashi H, Tani K, et al. Neutrophil chemotactic factors in the respiratory tract of patients with chronic airway diseases or idiopathic pulmonary fibrosis. Am Rev Respir Dis 1992;145:85-91.

26 Car BD, Meloni F, Luisetti M, et al. Elevated IL-8 and MCP-1 in the bronchoalveolar lavage fluid of patients with idiopathic pulmonary fibrosis and pulmonary sarcoidosis. Am $\mathcal{F}$ Respir Crit Care Med 1994;149:655-9.

27 Tazi A, Nioche S, Chastre J, et al. Spontaneous release of granulocyte colony-stimulating factor (G-CSF) by alveolar macrophages in the course of bacterial pneumonia and sarcoidosis: endotoxin-dependent and endotoxinindependent G-CSF release by cells recovered by bronchoalveolar lavage. Am f Respir Cell Mol Biol 1991;4:140-7.

28 Watari K, Asano S, Shirafuji N, et al. Serum granulocyte colony-stimulating factor levels in healthy volunteers and patients with various disorders as established by enzyme immunoassay. Blood 1989;73:117-22. 